\title{
ЦИФРО-АНАЛОГОВЫЕ ПРЕОБРАЗОВАТЕЛИ
}

\section{ДЛЯ ПЕРЕДАЧИ ВЫСОКОКАЧЕСТВЕННОГО ВИДЕОСИГНАЛА}

\author{
А.Лиль, Е.Иванова, И.Попов ${ }^{1}$
}

УДК 621.397 .6

BAK 05.27.00

\author{
Передача аналогового видеосигнала - неотъемлемая часть \\ большинства современных мультимедийных систем, включая как \\ системы промышленного применения, так и компоненты бытовых \\ систем. Помимо прочих преимуществ, использование аналоговых \\ интерфейсов позволяет сохранить совместимость с широким \\ спектром устройств предыдущего поколения, лишенных цифровых \\ интерфейсов.
}

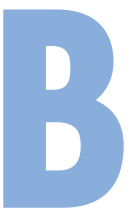

большинстве устройств современной бытовой техники видеосигнал обрабатывается в цифровом формате. Для передачи цифрового видеосигнала посредством аналоговых интерфейсов требуется преобразование сигнала из цифровой формы в аналоговую. Эту задачу решают цифро-аналоговые преобразователи (ЦАП), как показано на рис.1.

На первый взгляд, выбор цифро-аналогового преобразователя для конкретного проекта - задача достаточно формальная: требуется задать частоту дискретизации (sampling rate), разрешение (resolution) и напряжение питания, а затем подобрать подходящий компонент из представленных на рынке. Однако протоколы передачи видеосигнала накладывают определенные ограничения и вводят специфические требования к блокам цифро-аналоговых преобразователей. Одна из отличительных особенностей современных ЦАП - универсальность, то есть способность поддерживать различные стандарты видеопотока при сохранении высокого качества сигнала и низкой себестоимости аппаратуры. С позиций снижения себестоимости среди основных параметров аналоговых компонентов для систем на кристалле (СнК) стоит отметить наименьшую возможную занимаемую на кристалле площадь и потребляемую

\footnotetext{
ilya.popov@synopsys.com
}

мощность при гибкости настроек компонента под конкретные требования протокола.

Компания Synopsys обладает уникальным опытом разработки аналоговых компонентов и располагает полным набором ІР-блоков для реализации различных протоколов в системе на кристалле. На протяжении более 15 лет компания занимает лидирующие позиции в таких сегментах аналоговой схемотехники, как ЦАП, АЦП, аудиопреобразователи, преобразователи видеосигнала, решения для сенсорных подсистем. Наряду с ІР-блоками в портфолио компании входят компоненты для логического тестирования и моделирования на системном уровне.

В данной статье мы постараемся привести краткий обзор требований к цифро-аналоговым преобразователям для протоколов передачи видеосигнала и остановимся на основных характеристиках ЦАП для мультимедийных систем на кристалле.

\section{СПЕЦИФИКАЦИИ ВИДЕОФОРМАТОВ} И СТАНДАРТОВ АНАЛОГОВОГО ВИДЕОСИГНАЛА Большинство мультимедийных пользовательских приложений должны работать с несколькими видеоформатами, включая видео стандартной четкости (PAL или NTSC), высокой и ультравысокой четкости, а также графические форматы с высоким разрешением (QSXGA). В силу эволюции этих форматов в направлении увели- 


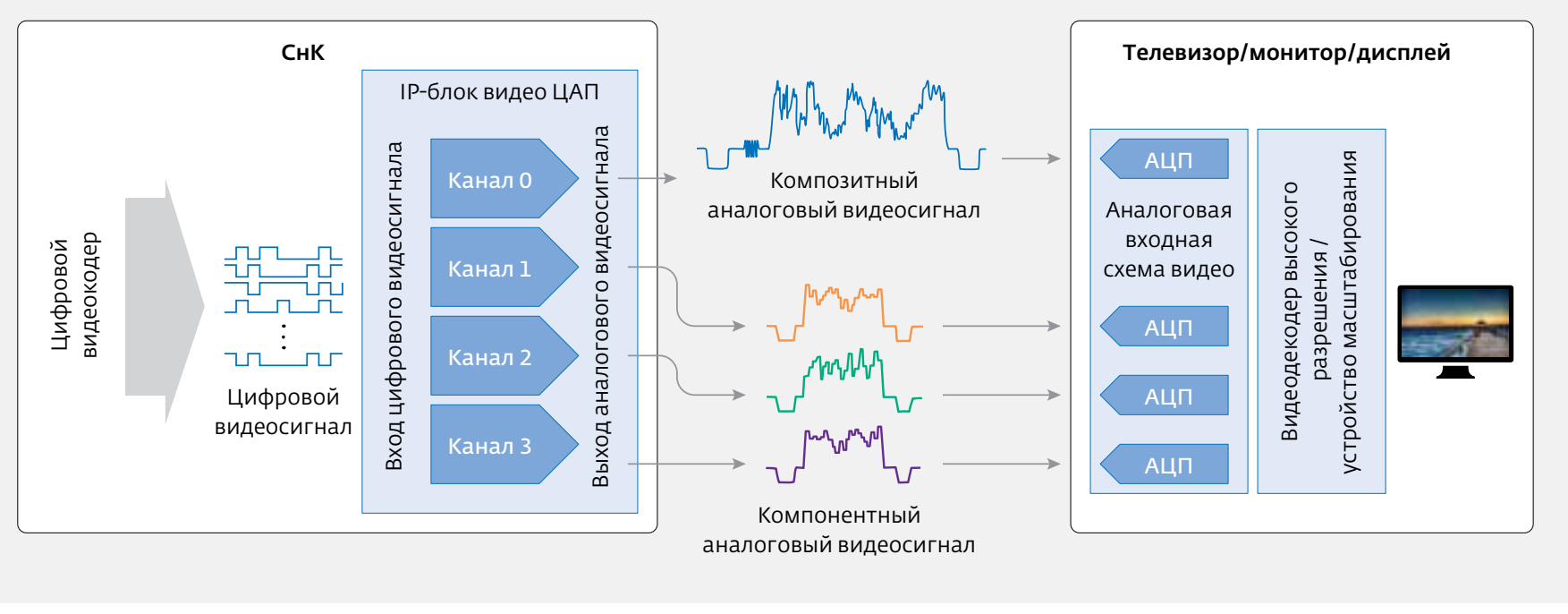

Рис.1. Преобразование цифровых видеосигналов в аналоговые с помощью видео ЦАП

чения полосы частот и частоты оцифровки требуется интеграция в современные мультимедийные СнК высокоскоростных видео ЦАП с частотой оцифровки выше 30 МГц (рис.2).

Обычно видеоинформация кодируется в компонентах яркости и цветности, к которым добавляются данные синхронизации местоположения на кадре и самого кадра в предопределенном формате, так чтобы приемник мог раскодировать данные о пикселе и синхронизировать полученное изображение. Форматирование видеосигнала стандартизовано для упрощения совместимости устройств. В настоящее время используются два основных способа передачи аналогового видеосигнала: композитный и компонентный.

Композитное видео (Color, Video, Blanking and Sync(VBS) - это формат аналогового видео, который передает полный видеосигнал по одному проводу. Этот формат может быть реализован различными способами в зависимости от способа кодирования цветности, размера кадра или частоты кадровой развертки. Например, в настоящее время в мире используются различные форматы: стандарт цветного телевидения Национального комитета по телевизионным стандартам NTSC (National Television Standards Committee), распространенный в странах Южной и Северной Америки, европейская система аналогового цветного телевидения PAL (Phase Alternating Line), являющаяся, по сути, развитием системы NTSC, и советско-французская система SECAM (Séquentiel couleur à mémoire). Компонентный видеосигнал представляет собой несколько параллельных каналов, передающих отдельные компоненты видеосигнала. Примером этого формата может служить RGB-стандарт, по которому каждый цвет (красный, зеленый, синий) передается по отдельному проводу.
В зависимости от типа видеосигнала - компонентного или композитного-амплитуда аналогового видеосигнала может достигать 1,3 В. На рис.3 показан пример композитного видеосигнала, сгенерированного видео ЦАП.

Стандарт видеосигнала VSIS Aссоциации по стандартам видеоэлектроники (VESA) описывает характеристики компонентного видеоформата для достижения целостности сигнала. Несоответствие влечет за собой деградацию качества изображения, проявляющуюся в таких параметрах, как насыщенность цвета, четкость контуров, размытость и зернистость. Стандарт VESA VSIS определяет электрические характеристики, такие как длительность переднего и заднего фронтов импульса, максимальная амплитуда, разрешающая способность, рассогласование каналов и чувствительность к шумовому сигналу.

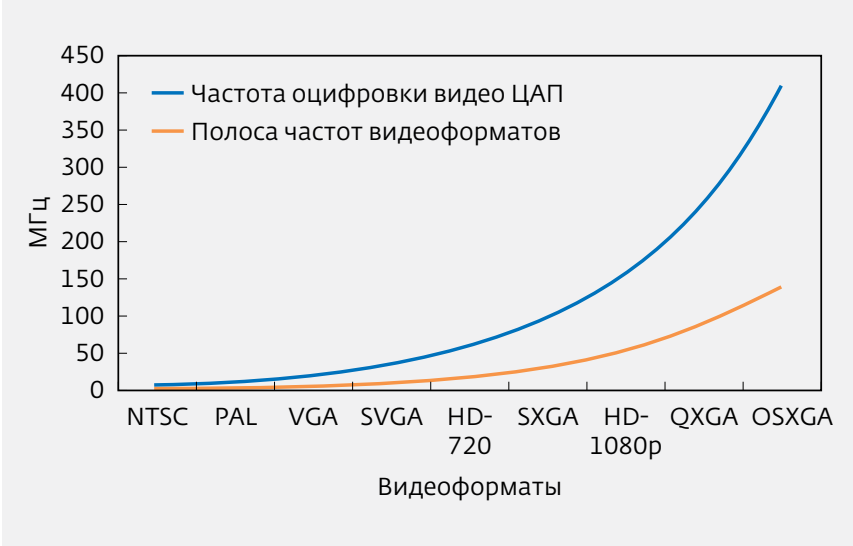

Рис.2. Эволюция полосы частот и частоты оцифровки видеоформатов и видео ЦАП 


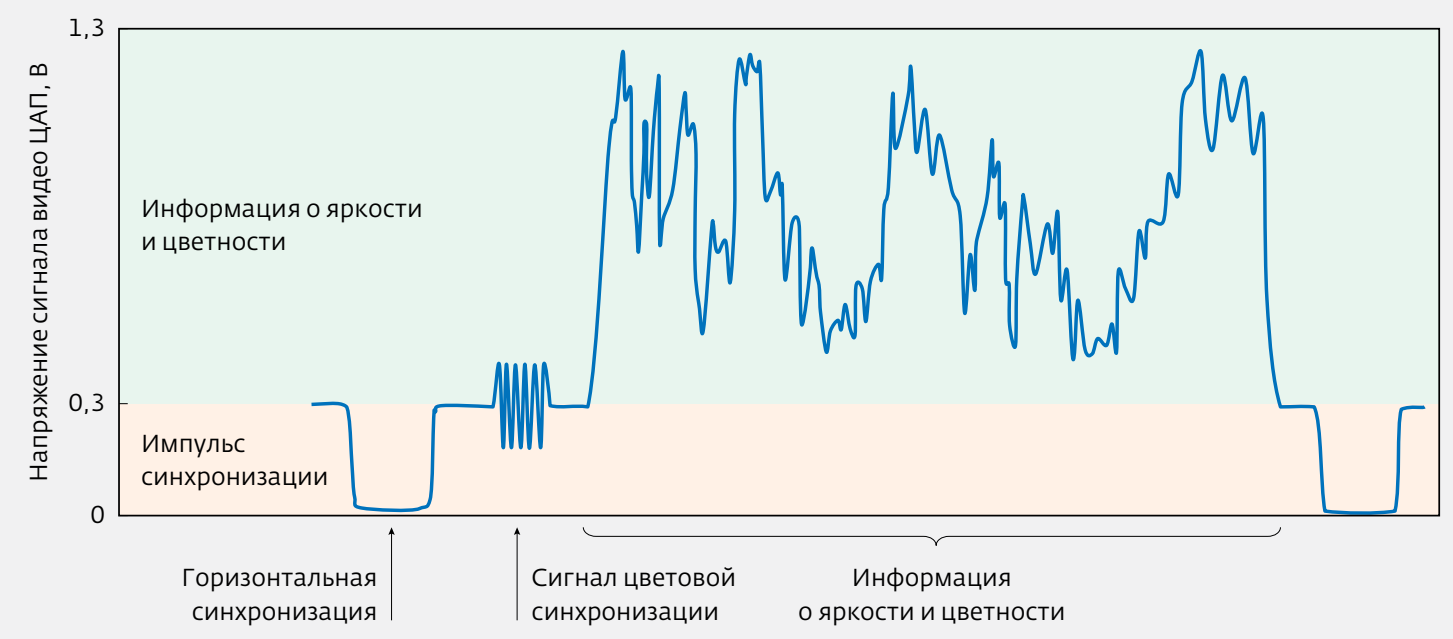

Рис.3.

Выходной композитный видеосигнал с видео ЦАП

\section{КЛЮЧЕВЫЕ ХАРАКТЕРИСТИКИ ВИДЕО ЦАП Совместимость со всеми видеоформатами и сигнальными стандартами}

Современные мультимедийные СнК реализуют комплексные функции обработки цифрового видео и совместимы с различными видеоформатами, упомянутыми выше. В свою очередь, видео ЦАП должен быть совместим с вышеуказанными стандартами и быть способен конвертировать различные цифровые видеосигналы, соответствующие разным видеоформатам, в высококачественный аналоговый сигнал.

\section{Архитектура ЦАП с суммированием токов}

Цифро-аналоговое преобразование видеосигнала требует высокого быстродействия и разрешающей способности и часто является ограничивающим фактором общей производительности системы. Архитектура с суммированием токов, обладая высокой скоростью

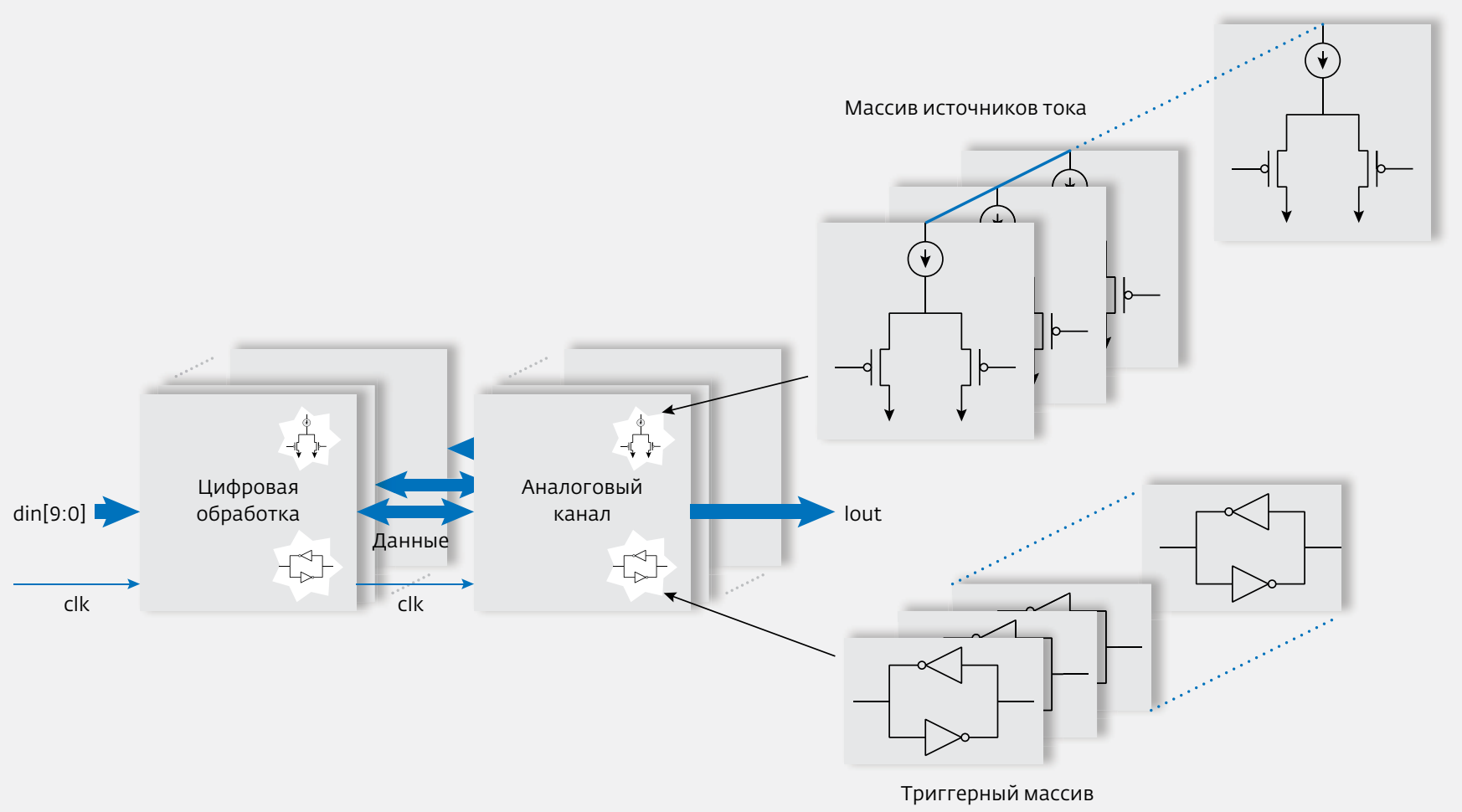

Рис.4. Архитектура ЦАП с суммированием токов 
и линейностью, способна удовлетворить требования современных видеоформатов. Возможность реализации данной архитектуры на продвинутых цифровых КМОП-технологиях делает ее идеальным решением для видео ЦАП. ЦАП на основе архитектуры с суммированием токов состоит из двух основных компонентов: массива согласованных источников тока и триггерного массива (рис.4).

Массив источников тока построен на КМОПтранзисторах. В отличие от токовых матриц на основе резистивных матриц, использование транзисторов в качестве источников тока значительно снижает рассеиваемую мощность. Для достижения пиковой производительности и сокращения нелинейности результирующего тока источники должны быть реализованы с использованием схемотехнических и топологических приемов, обеспечивающих минимальный разброс характеристик КМОП-транзисторов. В дополнение к этому точность передачи временных параметров тока между выводами должна хорошо контролироваться и при необходимости допускать подстройку. Массив симметричных триггеров, расположенный между декодирующей логикой и массивом источников тока, позволяет повысить точность временных параметров и снизить нелинейность и чувствительность к шумовым сигналам.
Архитектура с суммированием токов, построенная на КМОП-транзисторах, дает возможность снизить энергопотребление и работать со стандартными напряжениями технологических процессов 28 нм и ниже (например, 1,8 В для техпроцесса 28 нм). Это позволяет интегрировать данную архитектуру в систему на кристалле и снизить общую стоимость за счет отказа от дополнительных дискретных компонентов.

\section{Программируемое опорное напряжение}

Особенностью архитектуры ЦАП для видеопотока является необходимость поддержки видеоформатов с различной разрешающей способностью и, как следствие, с разными максимальными амплитудами выходного сигнала (рис.5).

Решение этой задачи возможно двумя способами: посредством цифровой обработки в целях сжатия изображения либо с помощью программируемого значения перепада выходного напряжения. Второй вариант предпочтительнее с точки зрения сохранения качества видеопотока.

\section{Генерация синхроимпульса}

В состав аналогового видеосигнала входят сигналы горизонтальной и вертикальной синхронизации (Hsync и Vsync), генерируемые цифровым видеокодером 


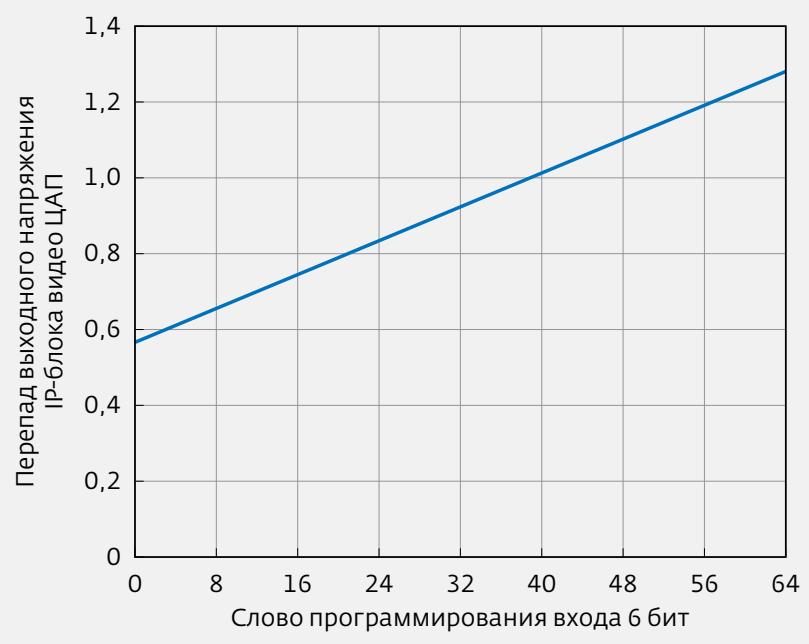

Рис.5. Пример диапазона программирования перепада выходного напряжения видео ЦАП

и передаваемые совместно с видеоданными по аналоговому каналу. Причем синхросигналы занимают нижний сегмент динамического диапазона. В примере, приведенном на рис.6, синхросигналы расположены в диапазоне 000h-124h или, иными словами, в нижней трети максимальной разности потенциалов для данного ЦАП (то есть, например, при максимальной разности потенциалов в 1 В величина синхросигнала составляет 0,285 В). Диапазоны синхросигналов и информации не перекрываются. Такое деление динамического диапазона позволяет отказаться от введения в систему дополнительного дискретного компонента для генерации отри- цательного синхроимпульса и, как следствие, снижает стоимость системы.

\section{Качественные характеристики ЦАП}

В композитном видеосигнале (используемом, например, в стандартах PAL, SECAM и NTSC) информация о яркости и цвете передается с помощью одного аналогового сигнала вкупе с синхроимпульсами.

Яркость модулируется посредством низкочастотной модуляции, а для передачи цвета используется фазовая модуляция несущей частоты. Несущая частота отличается в зависимости от выбранного стандарта - для NTSC она составляет 3,58 МГц, для PAL - 4,43 МГц. Стабильности фазы и несущей частоты являются основными качественными характеристиками цифро-аналогового преобразователя. Нестабильность несущей частоты или фазы вызывает изменения коэффициента усиления (Differential Gain, dG) или нестабильность фазы (Differential Phase, dP) соответственно. Это, в свою очередь, приводит к ухудшению качества видеосигнала в виде различий цветности и освещенности смежных пикселей со схожими характеристиками. Суммарный коэффициент нелинейных искажений (Total Harmonic Distortion, THD), представляющий собой отношение среднеквадратичного напряжения суммы высших гармоник сигнала, кроме первой, к напряжению первой гармоники,один из основных показателей качества выходного аналогового сигнала.

Интегральная нелинейность (INL) - это наибольшее отклонение уровня аналогового выходного сигнала от идеальной функции, измеряемое в шагах квантования (LBS). Совместное использование частотных (THD) и статических (INL) характеристик позволяет задать уровень качества цифро-аналогового преобразования (рис.7). Допустимым значением дифференциальной нелинейности независимо от используемого стандарта является значение, не превышающее шаг квантования ( \pm 1 LBS).

Нестабильность фазы, вызванная ресинхронизацией различных элементов токовой матрицы, вызывает изменение времени цикла между двумя синхросигналами (рис.8). Устойчивость к отклонениям фазы зависит от стандарта. Так, для обеспечения качественной передачи видеосигнала стандарта PAL разница между синхросигналами не должна превышать 1 пс.
Рис.6. Синхроимпульс, эквивалентный отрицательному импульсу 285 мВ, в динамическом диапазоне 10-битного видео ЦАП 


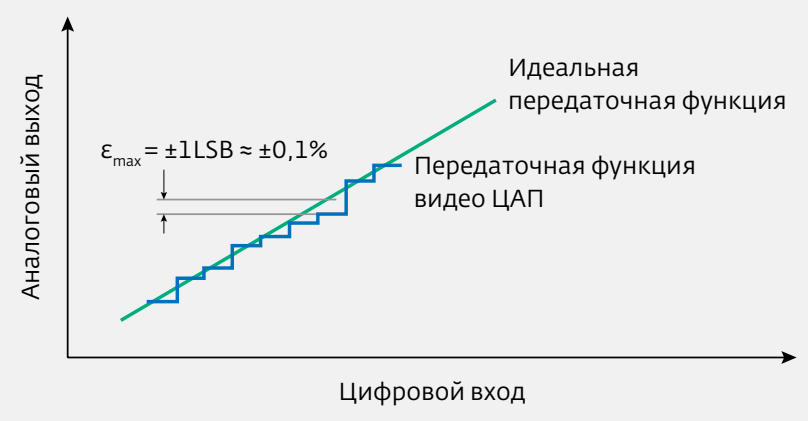

Рис.7. Передаточная функция 10-битного видео ЦАП с интегральной нелинейностью менее \pm 1 LBS

\section{Энергосбережение}

Для снижения энергопотребления система на кристалле способна определить неиспользуемые каналы, отсоединенные от приемника сигнала, и отключить соответствующий передатчик (рис.9).

Это достигается за счет дополнительной схемы, определяющей наличие нагрузки на дальнем конце линии передачи. Наличие подключенного приемника проверяется в интервалах между передачей основной видеоинформации и не влияет на характеристики видеопотока. При этом накладные расходы в случае правильного выбора нагрузки составляют незначительную величину. ЦАП, построенные на базе архитектуры с суммирова-

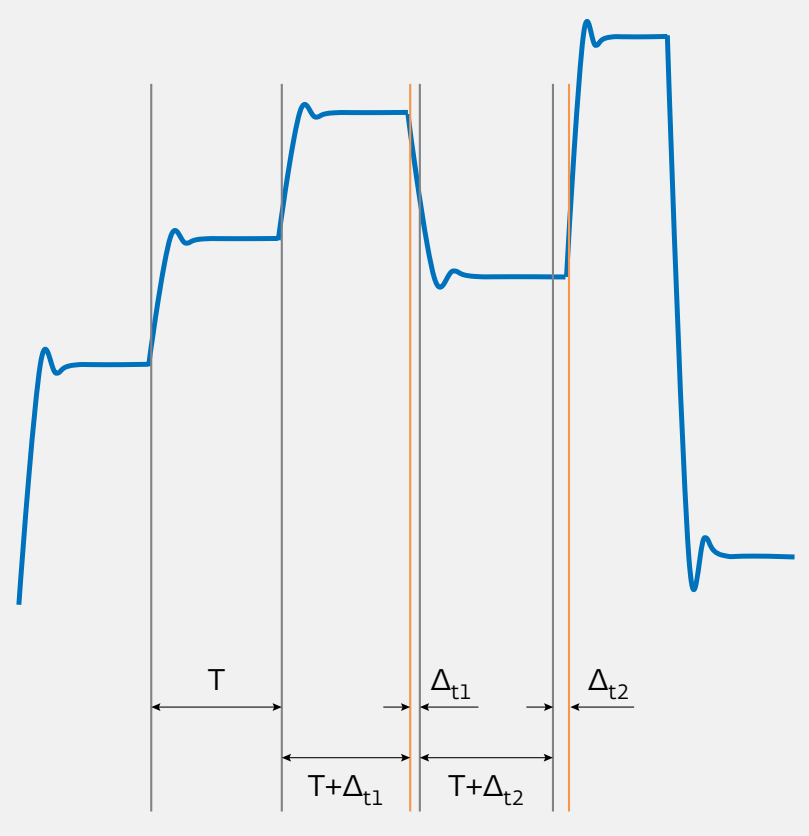

Рис.8. Временная неустойчивость выхода видео ЦАП

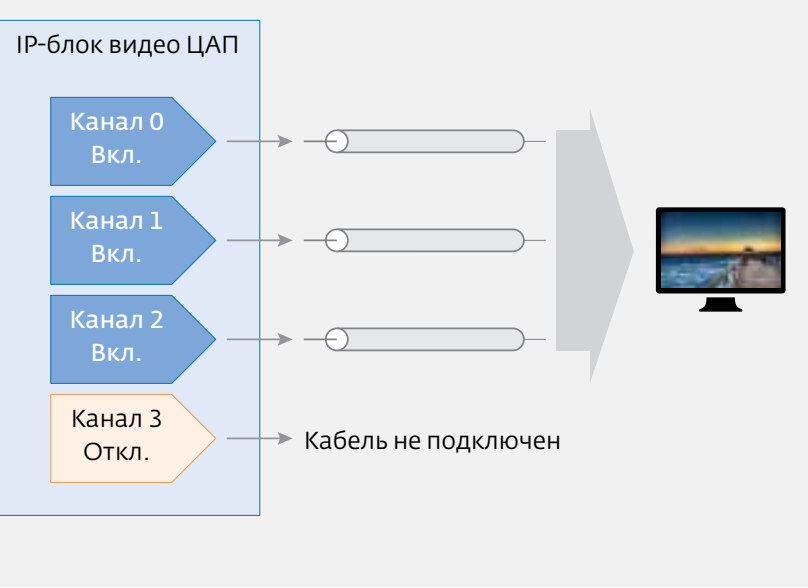

Рис.9. Экономия энергии в отключенных каналах в противоположность работающим каналам

нием токов, обладают высокой эффективностью и низкой рассеиваемой мощностью (рис.10).

\section{ИСПОЛЬЗОВАНИЕ \\ ВОССТАНАВЛИВАЮЩИХ ФИЛЬТРОВ}

Для стандартов передачи видео с частотой в несколько десятков мегагерц распространенной практикой является использование восстанавливающих фильтров на выходе ЦАП. Фильтры применяются для удаления гармоник исходного сигнала из результирующего спектра, возникающих в процессе дискретизации. Подобная фильтрация значительно повышает качество выходного сигнала и упрощает его дешифрацию на стороне приемника. Различные видеостандарты требуют использования фильтров с разными частотами среза в диапа-

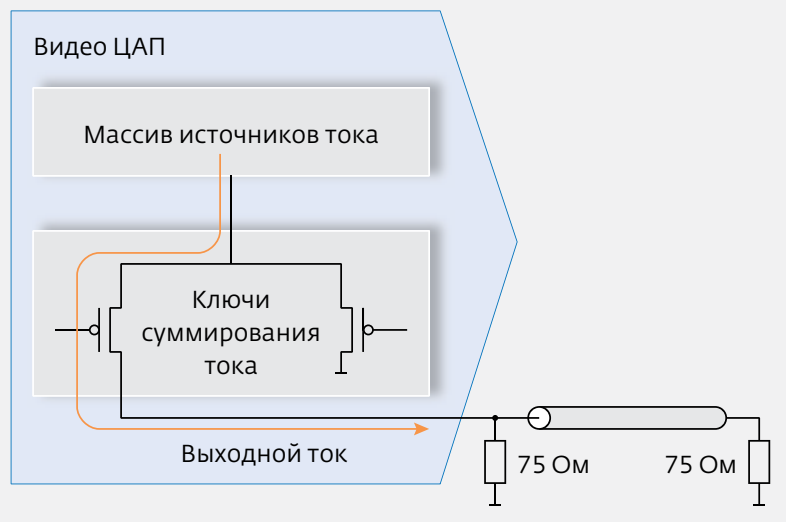

Рис.10. Ток, создаваемый массивом источников тока, передается в нагрузку на выходе 


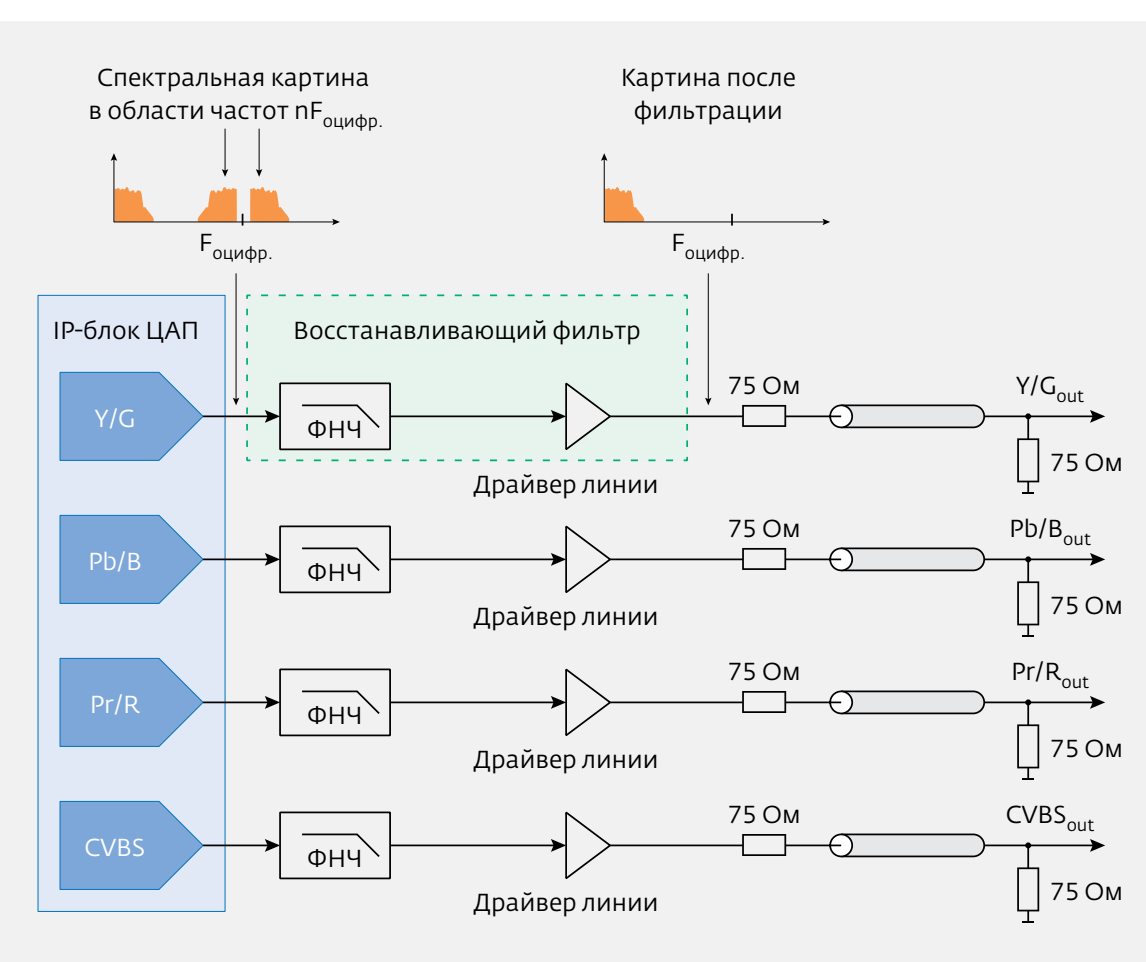

Рис.11. Фильтрация сигналов на выходе каждого видеоканала

Для создания необходимой амплитуды сигналов в согласованной таким образом линии ЦАП необходимо затратить 35 мА на каждый канал.

\section{ЗАЩИТА ОТ ЭЛЕКТРОСТАТИЧЕСКОГО РАЗРЯДА}

Любое устройство, подсоединенное к внешней линии передачи, подвержено опасности электростатического разряда (Electrostatic Discharge - ESD), величина которого может достигать 15 кВ. Данная проблема часто возникает при использовании аналоговых линий для связи удаленных камер с центром обработки сигнала или в сетях кабельного телевидения. Современные системы на кристалле устойчивы к электростатическому разряду порядка 2 кВ, что недостаточно для использования аналоговых линий, поэтому очевидно требуются

зоне от 10 МГц для эфирного телевидения стандартов PAL/SECAM/NTSC до 80 МГц для видеопотока высокого разрешения (рис.11).

Применение активных фильтров также играет значительную роль в снижении энергопотребления за счет отсечения в передающем канале паразитных гармоник, рассеивающих энергию. Для видеопотока сверхвысокого разрешения, такого как QSXGA (Quad Super Extended Graphics Array, $2560 \times 2048)$, восстанавливающие фильтры на передающей стороне не требуются. Однако для обеспечения передачи качественного изображения сверхвысокого разрешения линия должна быть сбалансирована как со стороны приемника, так и со стороны передатчика. Обычно используют сопротивления в 75 Ом с каждой стороны (рис.12).

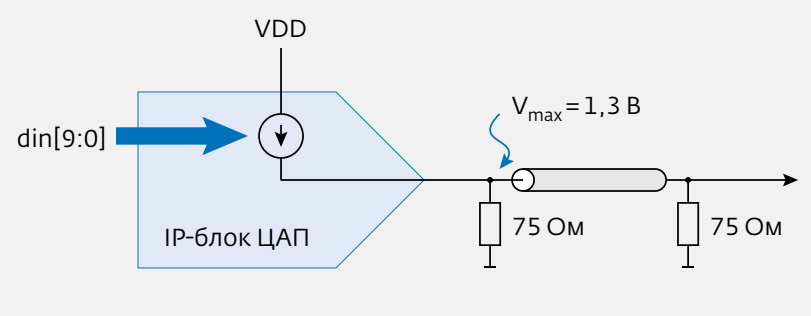

Рис.12. Видео ЦАП, работающий на видеокабель с терминаторами с двух сторон дополнительные меры защиты системы. Обычно для этой цели используется внешний блок, состоящий из двух обратносмещенных диодов между линией передачи и землей/питанием.

\section{ЗАКЛЮЧЕНИЕ}

Передача аналогового видеосигнала требуется в большинстве мультимедийных приложений. Для обеспечения цифро-аналогового преобразования цифровых видеосигналов различных стандартов к блокам ЦАП предъявляются жесткие требования по гибкости, производительности и энергопотреблению. На сегодняшний день архитектурой ЦАП, наиболее полно отвечающей требованиям современной мультимедийной системы на кристалле, является архитектура с суммированием токов (current steering), которая позволяет обеспечить требуемую разрядность и скорость преобразования для различных видеоформатов при использовании стандартной субмикронной КмОП-технологии. Создание качественного цифро-аналогового преобразователя требует использования схемотехнических и топологических приемов, обеспечивающих минимальный разброс характеристик КМОП-транзисторов с возможностью калибровки выходных параметров ЦАП. Возможность интеграции ЦАП в системе на кристалле по той же технологии, что и цифровой логики, позволяет снизить общую стоимость за счет отказа от дополнительных дискретных компонентов. 